\title{
The formation mechanism of membranes prepared from the crystalline EVAL polymer-water (nonsolvent)-2-propanol (nonsolvent) system
}

\author{
Tai-Horng Young ${ }^{*}$, Chih-Chen Hsieh, Li-Yen Chen, Yen-Shih Huang \\ Institute of Biomedical Engineering, College of Medicine and College of Engineering, National Taiwan University, Taipei 10016, Taiwan
}

Received 30 July 1998; received in revised form 16 October 1998; accepted 22 December 1998

\begin{abstract}
Crystalline poly(ethylene-co-vinyl alcohol) (EVAL) membranes from the water-2-propanol cosolvent were prepared by immersion-precipitation at $60^{\circ} \mathrm{C}$. The use of two nonsolvents serving as a cosolvent system, replacing the traditional solventnonsolvent pair, for the membrane formation was investigated. The two-nonsolvent proportion in which they are mixed to produce the cosolvent system and the prediction of the membrane structures were based on the complex ternary phase diagram at $60^{\circ} \mathrm{C}$. It was attempted to relate the influence of liquid-liquid demixing and solid-liquid demixing on the membrane morphologies to the ternary phase diagram. Two different morphologies and two different demixing rates could be obtained by using water and 2-propanol, respectively, to precipitate the cosolvent system. When water was used as the precipitation medium, it showed a rapidly precipitating system and cellular morphologies were obtained due to liquid-liquid demixing. In contrast, when 2-propanol was used as the precipitation medium, it showed delayed demixing and crystallization-induced particulate morphologies were formed. Trends expected on the basis of the phase diagram was in reasonable agreement with the observed membrane morphology. Therefore, the principles of membrane formation established for the ternary systems with nonsolvent-solvent-polymer can be extended to a nonsolvent-nonsolvent-polymer system. (C) 1999 Elsevier Science B.V. All rights reserved.
\end{abstract}

Keywords: Cosolvent; Crystallization; Liquid-liquid demixing; Phase diagram

\section{Introduction}

Most of the polymeric membranes that are in use are prepared via the immersion-precipitation process. This typical method involves casting a solution consisting of a polymer and a solvent on an appropriate substrate. Subsequently, the cast film is immersed in a nonsolvent as the precipitation medium to bring about

\footnotetext{
*Corresponding author. Tel.: +886-2-23970800; fax: +886-223940049; e-mail: thyoung@ ha.mc.ntu.edu.tw
}

phase separation to prepare the membrane. It has been recognized that liquid-liquid demixing and solidliquid demixing in a nonsolvent-solvent-crystalline polymer system play important roles in determining membrane structures. Liquid-liquid demixing results in the typical cellular morphology with pores from polymer-poor phase surrounded by the membrane matrix from polymer-rich phase. Solid-liquid demixing is from crystallization of regular segments of polymer to form membranes by linking of particles [1-7]. Aubert [1] studied the gelation of isotactic 
polystyrene by linking of growing particles. Lloyd et al. [2] studied the thermally induced phase separation process of isotactic polypropylene and polyethylene solutions. Bulte et al. [3] and Cheng et al. [4] prepared polyamide membranes with a spherulitic structure by solid-liquid demixing in the immersion-precipitation process. Witte et al. [5] did the same for poly-L-lactide with dioxane or $N$-methyl pyrrolidone as solvents and with methanol or water as nonsolvents. Therefore, for membranes fabricated from precipitation of crystallizable polymers, crystallization processes, other than liquid-liquid demixing, can also induce the membrane formation and are important for the membrane structure. Accordingly, the membrane structure may exhibit characters from both liquid-liquid demixing or/and solid-liquid demixing. In general, predictions of the membrane structures are by considerations of the thermodynamic (phase behavior) and kinetic (mass transfer) aspects of the immersion-precipitation process [7-14]. The relative locations of these two-phase separations in the phase diagram and the diffusion kinetics of the system will determine the importance of each demixing for the membrane morphology. Poly(ethylene-co-vinyl alcohol) (EVAL) membranes have been used as a nonsolvent-solvent-polymer model system for studies of the competition between liquid-liquid demixing and solid-liquid demixing during membrane formation in our laboratory [6,7,15-17]. In the work reported here, an alternative EVAL casting solution comprising of two liquids that individually are nonsolvents for the EVAL polymer was used to prepare membranes. Nonetheless, it is possible to mix these two nonsolvents, water and 2-propanol, in the appropriate volumetric proportions such that together they form a cosolvent system [16]. Therefore, mixing of two nonsolvents and polymer to form a cosolvent system represents a novel casting solution.

The equilibrium thermodynamics of the ternary system, water-2-propanol-EVAL, at $60^{\circ} \mathrm{C}$ has been described previously by using a ternary interaction parameter to investigate the cosolvency effect [16]. As shown in Fig. 1, it can be found that the phase behavior of EVAL-nonsolvent-nonsolvent system is more complex than that of traditional polymer-nonsolvent-solvent system. Two bimodals and one crystallization equilibrium curve divide the phase diagram into one single-phase region, one crystallization

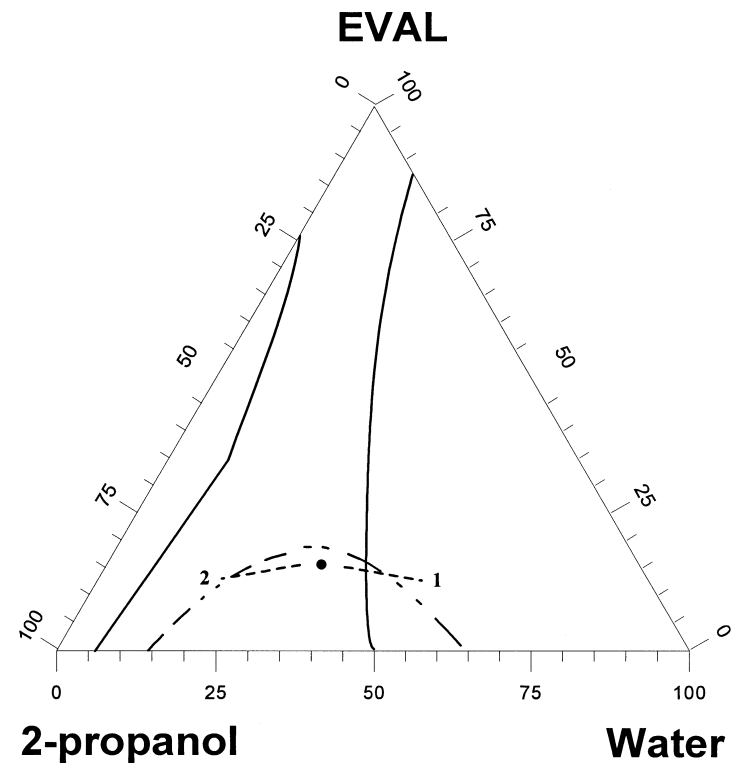

Fig. 1. Phase diagram of EVAL-water-2-propanol at $60^{\circ} \mathrm{C}$ [16]. ( $\_$: computed binodal; $\_$-._ : computed crystallization equilibrium line.) Any composition, above the crystallization curve, is metastable with respect to pure EVAL. The two regions between binodal and nonsolvent apex are metastable with respect to liquid-liquid phase separation. Therefore, the phenomenon of cosolvency will be located below the crystallization equilibrium curve and between the two binodal boundaries. A composition in this region is a homogeneous solution. The initial solution composition is denoted by the symbol $(\cdot)$. Curves 1 and 2 are the qualitative coagulation paths of membranes prepared from precipitating in water and 2-propanol, respectively.

region, one liquid-liquid demixing region and two overlapping regions that are metastable with respect to both liquid-liquid demixing and crystallization [16]. If excess water or 2-propanol was added to the singlephase region of the cosolvent system, then the solution exists from the solubility envelope and the mixture of water and 2-propanol no longer act as a cosolvent for the polymer. Therefore, this work was performed to elucidate the membrane formation mechanism that are responsible for the membrane structure from a cosolvent system. The attention is focused primarily on the correlation between the phase diagram and the membrane structure. The morphology and the crystallinity of the membranes have been evaluated using scanning electron microscopy (SEM) and differential scanning calorimetry (DSC), respectively. The objective of this contribution is to investigate whether the principles established for the traditional ternary systems with 
nonsolvent-solvent-polymer can be extended to a nonsolvent-nonsolvent-polymer system.

\section{Experimental}

\subsection{Material}

Membranes were prepared by using EVAL polymer (105A, Kuraray, Japan) having an average ethylene content of $44 \mathrm{~mol} \%$. LC-grade 2-propanol (Alps Chem, Taiwan) was used as received. Water was deionized and double distilled before use.

\subsection{Membrane preparation and characterization}

Membranes were prepared using the direct immersion-precipitation method. An appropriate amount of EVAL was dissolved in a cosolvent containing 40 vol\% water and 60 vol\% 2-propanol to form a $18 \mathrm{wt} \%$ EVAL homogeneous solution at $60^{\circ} \mathrm{C}$. This solution was dispersed uniformly on a glass plate (ca. $100 \mu \mathrm{m}$ ), and then immersed into the water and 2propanol bath to form membranes, respectively. In order to investigate the correlation between phase diagram and membrane morphology, the glass plate and the precipitation medium were kept at the same temperature $\left(60^{\circ} \mathrm{C}\right)$ as the polymer solution. Freezedried samples of the membranes were examined using SEM to obtain the membrane structures. In addition, the thermal property of EVAL membranes were studied using DSC (TA 2910, TA Instruments, USA) at the rate of $10^{\circ} \mathrm{C} / \mathrm{min}$.

\subsection{Measurement of precipitation time}

Light transmission experiments were performed to measure the time of the onset of precipitation due to phase separation in the membrane solution. A collimated light beam was directed to the membrane solution immersed in a nonsolvent bath. The light transmittance is recorded with a data acquisition system. The light intensity profiles are plotted as a function of time. The initial precipitation time is identified as the time at which transmission intensity starts to decrease. For detailed experimental setup and procedures, one can refer to the work of Reuvers et al. [12].

\section{Results and discussion}

\subsection{Membrane morphology}

The EVAL membrane, prepared by using water as the precipitation medium, shows an asymmetric structure consisting of a dense skin in the top surface and a thick porous support that is occupied by cellular morphologies with some macrovoids, as indicated in Fig. 2. Typical morphology of polymer crystallization (i.e., particulate) as discussed in previous reports [1-7] is not evident in this membrane. Therefore, the phase separation process responsible for this membrane is liquid-liquid demixing. Conversely, the membrane, prepared by using 2-propanol as the precipitation medium, shows that the morphology is composed of particles with a diameter of approximately submicron order, as indicated in Fig. 3. The particle structure is representative of crystallization from a homogeneous mixture [1-7]. No differences were observed between the morphologies of the top layer and the cross section, suggesting that all of the particles are appeared from individual EVAL nuclei born roughly simultaneously. Qualitative comparisons of Fig. 2 with Fig. 3 indicate that the membrane precipitated in water has an asymmetric structure, while the connectedness of the membranes precipitated in 2-propanol arises from surface contact between particles.

\subsection{The relation between cosolvency and membrane formation}

In this paragraph, the membrane formation from a cosolvent system is discussed by taking into account the characteristics of the cosolvency property. Although water and 2-propanol are nonsolvents for EVAL, water and 2-propanol will dissolve EVAL when these two nonsolvents are mixed together in certain proportions. We have proposed the increase in excess free energy of the water-2-propanol complex, the formation of intermolecular hydrogen bonding between this complex and vinyl alcohol segments of EVAL and this complex presenting a nonpolar environment in the vicinity of the ethylene segments of EVAL caused the phenomenon of cosolvency [16]. Therefore, when enough water-2-propanol contacts exist in the mixture, EVAL is able to form the max- 


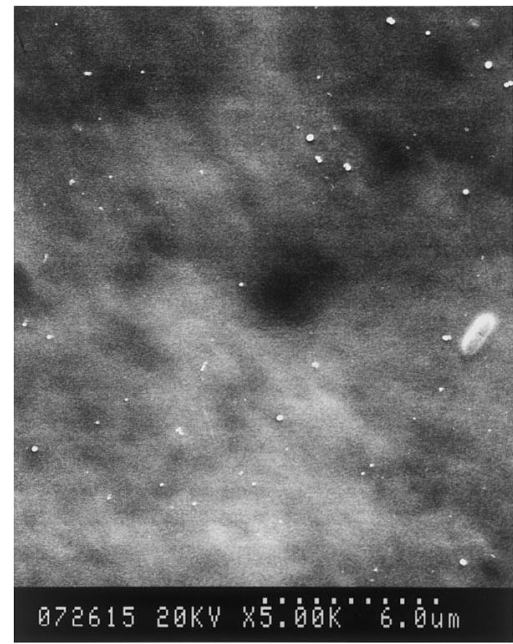

(a)

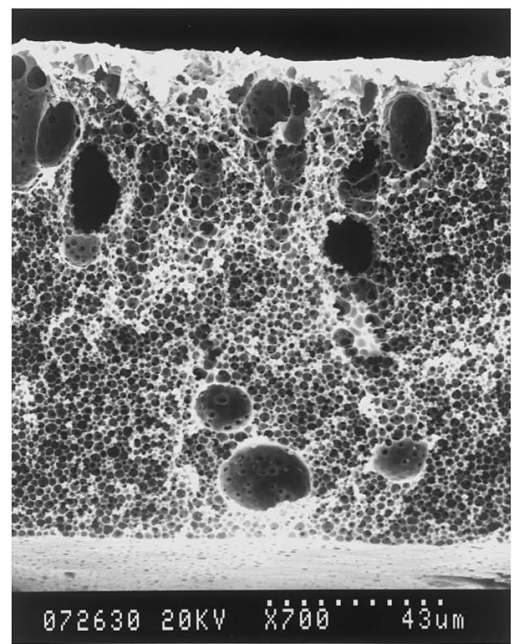

(b)

Fig. 2. The SEM photomicrographs of a membrane prepared by immersing a $18 \mathrm{wt} \%$ EVAL solution in a water bath: (a) top surface; (b) cross section.

imum number of water-2-propanol-EVAL contacts and will be expanded to the fullest extent allowed by these thermodynamic interactions. Even if two nonsolvents are against a given polymer when they are used separately, a mixture consisting of these two nonsolvents sometimes acts as a cosolvent to dissolve the polymer.

Overall, dissolution of the EVAL polymer will occur when a sufficient number of water-2-propanol contacts have formed to allow EVAL to be drawn into

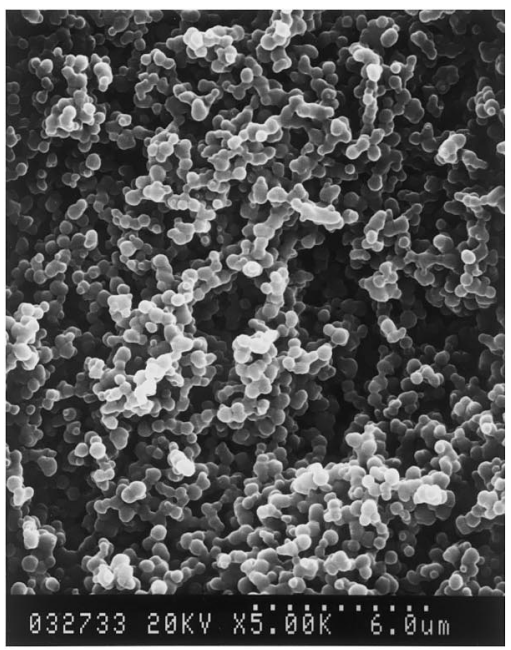

(a)

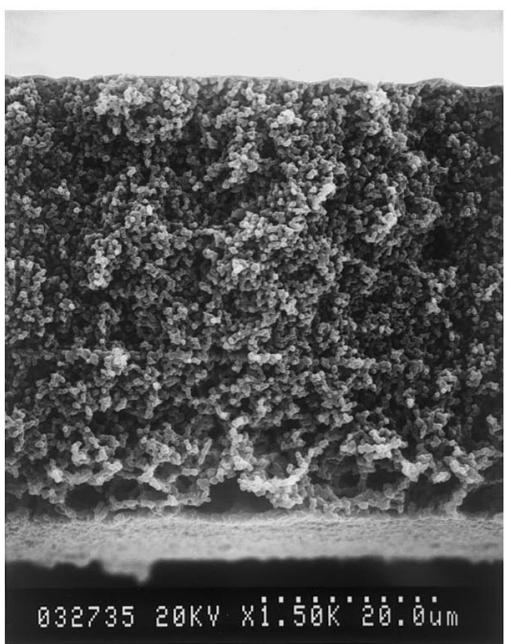

(b)

Fig. 3. The SEM photomicrographs of a membrane prepared by immersing a $18 \mathrm{wt} \%$ EVAL solution in a 2-propanol bath: (a) top surface; (b) cross section.

solution to reduce the net free energy of the system. However, when the polymer solution is immersed in water or 2-propanol, a change in the solvent composition reduces the water-2-propanol contacts. As neither water-EVAL nor 2-propanol-EVAL contacts are energetically favorable, any alteration in the solvent composition, which increases either water or 2-propanol in the mixture, will destroy the stability of water-2propanol-EVAL contacts. Therefore, more favorable water-2-propanol-EVAL contacts become less and 
the polymer begins to associate to a greater extent with other polymer molecules. When the system is no longer possible to maintain sufficient water-2-propanol-EVAL contacts, the EVAL solution demixes to form membranes.

In this work, the effect of addition of water or 2propanol to the EVAL solution on the phase separation was different. Water acting as a precipitation medium induces a liquid-liquid demixing. Conversely, the phase separation process yields small crystalline particles when 2-propanol acts as a precipitation medium. Therefore, the phase separation during EVAL membrane formation resulting from adding different nonsolvents will have a great effect on the final membrane structure. We conjecture that such remarkable differences between the effects of water and 2-propanol are caused by differences of individual molecular properties, for example the electric state, the molecular structure and so on. In particular, we hypotheses the effect of the interaction of the $\mathrm{CH}_{3}$ groups of 2propanol and the ethylene segments of EVAL cannot be ignored. However, it will be very difficult to understand the contribution of molecular individuality to the change in the membrane structure quantitatively. Therefore, we discuss the membrane formation mechanism by qualitatively superimposing the coagulation paths on the entire thermodynamic phase diagram as shown in Fig. 1. The point shown as a filled circle $(\bullet)$ denotes the initial composition of the casting solution prepared by using water-2-propanol cosolvent to prepare membranes. The coagulation paths of membranes prepared from precipitating in water and 2-propanol are denoted by curves 1 and 2, respectively. Since water and 2-propanol locate on the opposite sides of the initial casting solution, the coagulation path of membrane formation will move toward the nonsolvent side in the phase diagram, but in two different directions. It can be found that water denoted by 1 brings the membrane solution into a metastable state initially with respect to liquid-liquid demixing, and then with respect to solid-liquid demixing. In other words, it is expected that the membrane should exhibit a typical cellular morphology due to liquid-liquid demixing. Hence, the membrane structure is consistent with the prediction on the basis of the thermodynamic viewpoint. By contrast, the coagulation path of membrane precipitated in 2-propanol denoted by 2 is first metastable with respect to solid-liquid demixing. However, crystallization is a slow process in comparison with liquid-liquid demixing [18] because of the time needed for orientation of the polymer molecule, both for nucleus formation and for growth. This suggests that although crystallization has the opportunity to take place prior to liquid-liquid demixing, precipitation in the form of crystallization must occur in a such slow rate to form crystallization-induced structure that it begins prior to liquid-liquid demixing or at least both crystallization and liquid-liquid demixing occur simultaneously [7]. Therefore, we can anticipate that the time of the membrane formation for 2-propanol as the precipitation medium must be long enough to proceed solid-liquid demixing, which is shown in Section 3.3.

\subsection{Light transmission}

Light transmission experiments were performed to measure the time of the onset of precipitation of the membrane solution in the precipitation bath. The principle of light transmission experiments is that the light transmittance of the membrane solution would decrease with the appearance of optical inhomogenieties, which can be induced by liquid-liquid demixing or solid-liquid demixing. Therefore, the time that the light transmittance begins to drop can be used to predict the rate of phase separation during membrane formation. The results of light transmission experiments for water and 2-propanol as the precipitation media are shown in Fig. 4. For each profile, the time at which light intensity begins to decrease is identified as the onset point of precipitation. Clearly, the instantaneous demixing takes place for water as the precipitation medium. Accordingly, the binodal was crossed right after immersion of EVAL solution to induce liquid-liquid demixing. By contrast, it indicates a measured precipitation time of approximately $30 \mathrm{~s}$ for 2-propanol as the precipitation medium, significantly longer than the instantaneous demixing case. Because of such postponement for the membrane formation process, nucleation of crystallites within the membrane solution becomes feasible [8]. As discussed above, the mechanism of phase separation will be significantly different for using different nonsolvents and it will significantly adjust the resulting membrane structure. 


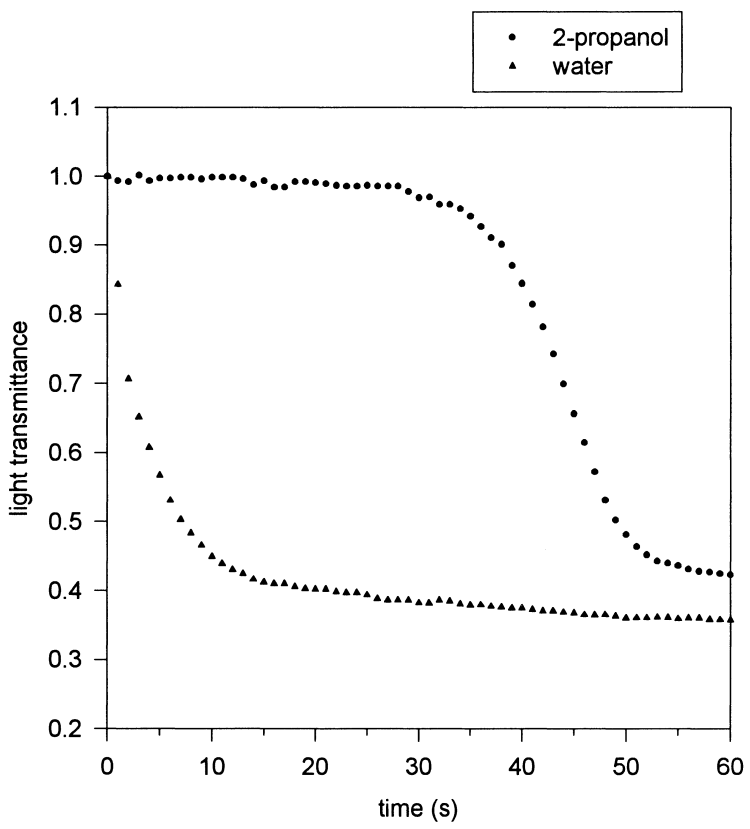

Fig. 4. Light transmission for precipitation of a $18 \mathrm{wt} \%$ EVAL solution in water and 2-propanol baths.

\subsection{Characterization of EVAL membranes by DSC}

Evidence of crystallization during membrane formation can be found in the DSC thermograms, as shown in Fig. 5. It appears that both EVAL membranes have a melting peak at $165^{\circ} \mathrm{C}$ and enthalpy of fusion (about $45 \mathrm{cal} / \mathrm{g}$ ) similar to that of the original EVAL pellet specimen. This suggests that crystallinity of the polymer maintains in the present precipitation process. Although liquid-liquid demixing may dominate the cellular morphology for EVAL solution precipitated in water, crystallization actually appears in the nascent membrane after liquid-liquid demixing. As shown in Fig. 1, this can be explained that the polymer-rich phase falls into the crystallization region after liquid-liquid demixing has occurred. Therefore, the liquid-liquid demixed structure is followed and stabilized by crystallization. However, crystallization only has a small effect on the overall morphology of the formed membrane because it occurs after growth of pores and the wall of pores will be fixed. On the other hand, if crystallization takes place prior to liquid-liquid demixing during the membrane formation for EVAL membranes precipitated in 2-propanol,

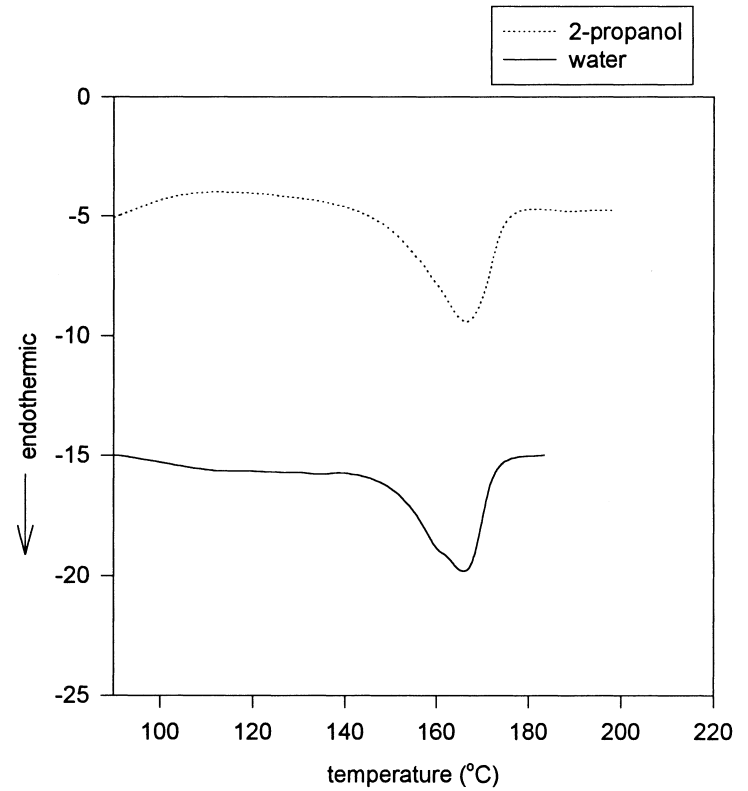

Fig. 5. DSC melting endotherms of EVAL membranes prepared by immersing a $18 \mathrm{wt} \%$ EVAL solution in water and 2-propanol baths at the heating rate of $10^{\circ} \mathrm{C} / \mathrm{min}$.

EVAL molecules will form particles distributing in the membrane. Nonetheless, it is impossible, for these particles are clustered together, to reorganize their distribution from within their domains to form cellular pores or macrovoids, i.e., particles will be fixed in their location to suppress the tendency of liquid-liquid demixing [17].

\section{Conclusion}

EVAL membranes from the water-2-propanol cosolvent were prepared by immersion-precipitation at $60^{\circ} \mathrm{C}$. Once the cast solution is immersed into water or 2-propanol, phase separation of polymer solution takes over the cosolvency to make the membrane. Depending upon the sequence of the phase separation during precipitation processes, the formed membranes may exhibit cellular and particulate morphologies. If the precipitation medium is water, liquid-liquid demixing dominates the membrane formation mechanism and an asymmetric cellular morphology is formed. For the case of immersing in 2-propanol, crystallization may precede liquid-liquid demixing 
and a particulate morphology will form. Therefore, the formation of membrane structure from different nonsolvents is induced by different phase transition. In this work, a good correlation between phase diagram and morphology was found. The different membrane morphologies can be clearly represented through the use of the phase behavior of a cosolvent system at $60^{\circ} \mathrm{C}$.

\section{Acknowledgements}

The authors thank the National Science Council of the Republic of China for their financial support of project NSC 86-2216-E-002-003.

\section{References}

[1] J.H. Aubert, Isotactic polystyrene phase diagrams and physical gelation, Macromolecules 21 (1988) 3468-3473.

[2] D.R. Lloyd, K.E. Kinzer, H.S. Tseng, Microporous membrane formation via thermally induced phase separation I. Solidliquid phase separation, J. Membr. Sci. 52 (1990) 239-261.

[3] A.M.W. Bulte, B. Folkers, M.H.V. Mulder, C.A. Smolders, Membranes of semicrystalline aliphatic polyamide nylon 4,6: formation by diffusion-induced phase separation, J. Appl. Polym. Sci. 50 (1993) 13-26.

[4] L.P. Cheng, A.W. Dwan, C.C. Gryte, Membrane formation by isothermal precipitation in polyamide-formic acid-water systems I. Description of membrane morphology, J. Polym. Sci., Polym. Phys. 33 (1995) 211-222.

[5] P. van de Witte, H. Esselbrugge, P.J. Dijkstra, J.W.A. van de Berg, J. Feijen, A morphological study of membranes obtained from the systems polylactide-dioxane-methanol, polylactide-dioxane-water, and polylactide- $N$-methyl pyrrolidone-water, J. Polym. Sci., Polym. Phys. 34 (1996) 25692578.

[6] T.H. Young, J.Y. Lai, W.M. Yu, L.P. Cheng, Equilibrium phase behavior of the membrane forming water-DMSOEVAL copolymer system, J. Membr. Sci. 128 (1997) 55-65.
[7] L.P. Cheng, T.H. Young, Y.M. You, Formation of crystalline EVAL membranes by controlled mass transfer process in water-DMSO-EVAL copolymer systems, J. Membr. Sci. 145 (1998) 77-90.

[8] F.W. Altena, C.A. Smolders, Calculation of liquid-liquid phase separation in a ternary system of a polymer in a mixture of a solvent and a nonsolvent, Macromolecules 15 (1982) 1491-1497.

[9] L. Yilmaz, A.J. McHugh, Analysis of nonsolvent-solventpolymer phase diagrams and their relevance to membrane formation modeling, J. Appl. Polym. Sci. 31 (1986) 9971018.

[10] C. Cohen, G.B. Tanny, S.J. Prager, Diffusional-controlled formation of porous structures in ternary polymer systems, J. Polym. Sci., Part A-2 17 (1979) 477-489.

[11] L. Yilmaz, A.J. McHugh, Modeling of asymmetric membrane formation I. Critique of evaporation models and development of a diffusion equation formalism for the quench period, J. Membr. Sci. 28 (1986) 287-310.

[12] A.J. Reuvers, J.W.A. van der Berg, C.A. Smolders, Formation of membranes by means of immersion precipitation. Part I. A model to describe mass transfer during immersion precipitation, J. Membr. Sci. 34 (1987) 45-65.

[13] P. Radov, S.W. Thiel, S.-T. Hwang, Formation of asymmetric polysulfone membranes by immersion precipitation. Part I. Modeling mass transport during gelation, J. Membr. Sci. 65 (1992) 213-229.

[14] L.P. Cheng, A.W. Dwan, C.C. Gryte, Membrane formation by isothermal precipitation in polyamide-formic acid-water systems II. Precipitation dynamics, J. Polym. Sci., Polym. Phys. 33 (1995) 223-235.

[15] T.H. Young, L.P. Cheng, W.M. You, L.Y. Chen, Prediction of EVAL membrane morphologies using the phase diagram of water-DMSO-EVAL at different temperatures, Polymer. Sci. 40 (1999) 2189-2195.

[16] T.H. Young, L.P. Cheng, C.C. Hsieh, L.W. Chen, Phase behavior of EVAL polymers in water-2-propanol cosolvent, Macromolecules 31 (1998) 1229-1235.

[17] T.H. Young, D.M. Wang, C.C. Hsieh, L.W. Chen, The effect of the second phase inversion on microstructures in phase inversion EVAL membranes, J. Membr. Sci. 146 (1998) 169178.

[18] A.J. Reuvers, F.W. Altena, C.A. Smolders, Demixing and gelation behavior of ternary cellulose acetate solutions, J. Polym. Sci., Polym. Phys. 24 (1986) 793-804. 